Article

\title{
The Transition from Static to Dynamic Boundary Friction of a Lubricated Spreading and a Non-Spreading Adhesive Contact by Macroscopic Oscillatory Tribometry
}

\author{
Christof Koplin *, Sherif Ahmed Abdel-Wahed, Raimund Jaeger and Matthias Scherge \\ Fraunhofer IWM MikroTribologie Centrum, Wöhlerstr. 9, 79108 Freiburg, Germany; \\ Sherif_abdelwahed@hotmail.com (S.A.A.-W.); raimund.jaeger@iwm.fraunhofer.de (R.J.); \\ matthias.scherge@iwm.fraunhofer.de (M.S.) \\ * Correspondence: christof.koplin@iwm.fraunhofer.de; Tel.: +49-761-5142-269
}

Received: 20 November 2018; Accepted: 25 December 2018; Published: 9 January 2019

check for updates

\begin{abstract}
Lubricated poly(ether ether ketone) (PEEK) and polyamide (PA46)-steel tribosystems were investigated. They show a complex but systematic transition behavior from static to boundary friction, to dynamic friction or to mixed-lubrication. Nonstandard macroscopic oscillatory tribometry as well as gliding experiments were carried out. A previous study showed that the surface and interfacial energies of PEEK, lubricant and steel can indicate trends in the tribological behavior. In the current study, these findings are confirmed for PA46 and a wider range of lubricants. It was shown that a reversal of the order of the work of spreading of two lubricants by switching from PEEK to PA46 as polymer component in the tribological system also resulted in a reversal of the coefficient of friction (COF) at low gliding velocities for these systems. The adhesion threshold of PA46 with the non-spreading lubricants water, glycerine, a water-glycerine mixture, ethylene glycol and poly-1-decene decreased with increasing solving tendency of the lubricants in contrast to the previous results for spreading lubricants for PEEK. Furthermore, the onset of forced wetting was studied for lubricants with different surface and interfacial energies and viscosities $\eta$. In general, a $1 / \eta$ dependency was observed for the velocity which marks the onset of forced wetting. This agrees with theoretical models.
\end{abstract}

Keywords: low velocity friction; rate-and-state friction; spreading; forced wetting; squeeze out

\section{Introduction}

Tribologically loaded components that are used in mechanical engineering or in the automotive industry can be made from thermoplastic polymers in order to obtain light weight and energy efficient products or components which exhibit a superior performance with respect to noise, vibration and harshness. The use of polymers in tribological applications requires however a profound understanding of the friction and wear behavior of polymers [1]. In particular the tribology of lubricated polymer-steel contacts is complex and remains an area of active research. The complexity partially arises from the interaction of polymer, lubricant and the steel frictional partner. The observation of polymer transfer [2] and the "temperature hot spots" [3] of the polymer during frictional loading in lubricated systems indicate that adhesive friction contributes to the tribology of polymers. Physico-chemical interactions between the frictional partners play an important role in particular in the area "left of the Stribeck curve" [4], i.e., in the boundary friction regime. In turn, experimental techniques which focus on the tribological behavior in the boundary friction regime can 
yield some insight into the influence of the physico-chemical characteristics of the frictional partners on the tribology of the system.

In a recent study [5], the transition from static to dynamic boundary friction of lubricated poly(ether ether ketone) (PEEK)-steel contacts was investigated with oscillatory tribometry and the recording of Stribeck curves ranging from very low to moderate gliding velocities. The tribological measurements were accompanied by measurements of the surface and interfacial energies of the tribological partners. The results of this study suggest that differences in the tribological behavior that were observed for two different ester-based lubricants can be explained by differences in the surface and interfacial energies. The system where the lubricant has a higher tendency of spread into the tribological contact exhibits a lower coefficient of friction at very low gliding speeds. The system where the lubricant showed a stronger tendency to "solve" the polymer surface and, thus, make the polymer chains near the surface more mobile, shows an enhanced adhesive friction and prolonged stiction-phase prior to the onset of gliding.

In the current paper, we want to investigate whether or not the approach to explain the tribological behavior of lubricated polymer-steel systems with surface and interfacial energies is feasible for a broader range of polymers and lubricants. In addition to PEEK, a thermoplastic polymer with higher polar component of the surface energy, poly(amide) 46 (PA46), was studied. The lubricants included in addition to the previously investigated pentaerythrite ester (PEEs) and trimellitic acid ester (TAEs) were poly(1-decene) as a non-polar lubricant, and water, glycerine and ethylene glycol as polar lubricants. With this selection of polymers and lubricants, a situation where no spontaneous spreading of the lubricant into the tribological contact occurs can be investigated. We can therefore address the question of whether or not the findings of the previous study [6] on spreading systems also apply for non-spreading systems: do we observe a correlation between the coefficient of friction (COF) at low gliding velocities and the magnitude of the work $W_{\text {spreading, }}$ which describes the tendency of the lubricant to enter the tribological contact? Does the adhesion threshold measured in the oscillatory tribometry also correlate for non-spreading systems with the magnitude of the work $W_{\text {solving, }}$ which was interpreted in the previous paper [6] as the tendency of the lubricant to enter into the surface layer of the polymer and to mobilize the chains?

In particular, we want to test whether or not the order of the COF at low gliding velocities

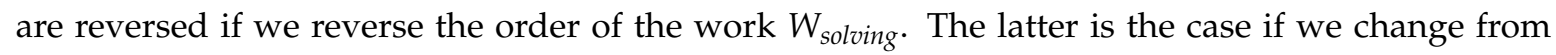
a PEEK-steel tribosystem to a PA46-steel tribosystem if PEEs and TAEs are used as lubricants. Furthermore, would a reversal of the COF also be observed if the non-spreading lubricants ethylene glycole and glycerine are used? The ranking of the spreading energies $W_{\text {spreading }}$ would suggest such a result for both cases.

In addition to the work presented in the previous study [5], we want to study the behavior of the tribological systems at higher gliding velocities. It is well established that the viscosity of the lubricant has a crucial effect on the frictional behavior of lubricated systems. The viscosity of water-glycerine mixtures can be varied over several orders of magnitude. By carrying out experiments with different water-glycerine mixtures we can potentially distinguish tribological regimes which are dominated by a forced wetting of the tribological contact and thus by the viscosity of the lubricant from tribological regimes that are dominated by the surface and interfacial energies of their constituents.

\section{Motivation and Scientific Background}

The experimental studies discussed in the current paper focus mainly on the effect of lubricants on the adhesive contact and the adhesive friction between thermoplastic polymers and a smooth steel frictional partner. The adhesive friction of polymers arises from the continuous forming and breaking of adhesive bonds between the two gliding partners. This process was described by Schallamach as a thermally driven rate process. Schallamach's model [6] for adhesive friction results in a coefficient of friction (COF) which initially grows monotonously with increasing gliding velocity. The COF reaches a maximum value at a specific gliding velocity and decreases if the gliding velocity is increased 
further. Schallamach's model was confirmed experimentally in a range of studies [7-9] and refined by, e.g., Singh et al. [10]. Since Schallamach's approach is based on an energy-based description of the slip rate for adhesive processes between the frictional partners (Equation (1)), their surface and interfacial energies can give some insight into the influence of the lubricant on the adhesive friction (Equations (2) and (4)). A good correlation was found for wetting and spreading parameter of lubricated self-pairing contacts for a wide range of lubricants [11,12].

Quantities like the work of spreading $W_{\text {spreading }}$ and the work of solving $W_{\text {solving }}$ can be calculated with surface and interfacial energies (Equations (5) and (6)). The determination of surface and interfacial energies of the frictional partners $W_{i j}$ is typically based on contact angle measurements $(i=1$ refers to the polymer, $i=2$ to the steel frictional partner and $i=3$ to the lubricant). The work of adhesion $W_{13}$ can be determined, for instance, with the Young-Dupree equation (Equation (3)) by measuring the contact angle between lubricant and polymer. An alternative approach described by Owens is to determine $W_{13}$ with suitable averages of the polar and dispersive components of the free surface energy $\gamma_{11}$ for the polymer and the cohesive energy $\gamma_{33}$ of the liquid, where the latter are also determined with contact angle measurements, but possibly by using several other liquid-solid combinations than the one for which the work of adhesion is calculated. If there is a strong interaction between the lubricant and the polymer (which is the case, e.g., for water-based lubricants and poly(amides)), both approaches can yield different results. The ratio of interfacial energy $\gamma_{13}$ determined by Owen's approach and by a contact angle measurement with the pairing of lubricant and polymer (denoted as $W_{\text {solving }}$ ) is called the "interaction parameter". An interaction parameter greater than unity indicates a strong interaction between polymer and lubricant. Equations (1)-(8) summarize the relevant mathematical expressions; for a detailed description of the approach we refer to an earlier publication [5].

slip rate of adhesive contact surface energy

contact angle equation

model for interfacial energy

spreading energy of system

solving energy of lubricated thermoplastic

interaction parameter

velocity for forced wetting

$$
\begin{gathered}
f=f_{0} e^{-\frac{E+W+\Omega}{k_{b} T}} \\
\gamma_{i i}=W_{i i} / 2 \\
\gamma_{33} \cdot(1+\cos \theta)=\gamma_{11}+\gamma_{33}-\gamma_{13}=W_{13} \\
W_{13}=2 \cdot \sqrt{\gamma_{11}^{p} \cdot \gamma_{33}^{p}}+2 \cdot \sqrt{\gamma_{11}^{d} \cdot \gamma_{33}^{d}} \\
W_{\text {spreading }}=W_{12}+W_{33}-W_{13}-W_{23} \\
W_{\text {solving }} \leq \gamma_{13}=1 / 2 W_{11}+1 / 2 W_{33}-W_{13} \\
W_{\text {solving }} / \gamma_{13} \\
v \sim W_{12} / \eta
\end{gathered}
$$

Schallamach's model for adhesive friction can describe the velocity dependence of the COF over a wide range of gliding velocities. At the transition from static friction to very low gliding velocities, additional effects have to be included in the interaction between the frictional partners in order to be able to explain the velocity dependence of the COF. For some systems, a COF which initially decreases with increasing gliding velocity is observed. This behavior was observed at the transition from static to dynamic friction, e.g., metal on metal, paper on paper and, in a recent study, for lubricated polymer on steel [5] tribological contacts. The variety of tribological systems suggests these systems do not share a common type of "microscopic mechanism" that causes the development of the COF at low velocities. The behavior at low velocities is governed by a rather universal process. The "rate-and-state" theory of Dieterich and Ruina takes the "state of the contact area" into account and assumes an aging process of the contact area $[13,14]$. The resulting friction laws exhibit an initial decrease of the COF with increasing sliding velocity, until a minimum COF is reached. Putelat et al. identified for several tribological systems the microphysical mechanisms which cause the aging process of the contac area [15]. 
The range of velocities for which Schallamach's model for adhesive friction can be applied also has a limit at high velocities for lubricated systems. When the gliding velocity of lubricated systems is increased further after the maximum of the $\mathrm{COF}$ is reached and the COF decreases in the mixed lubrication regime, a minimum and subsequent increase of the COF marks the onset of the hydrodynamic friction. The frictional partners are separated by the lubricant, and the viscous response of the lubricant results in increase of the COF with increasing sliding velocities. This behavior is also observed for non-spreading lubricants, i.e., for lubricants which from a purely energetic point of view would not enter the tribological contact. If a critical velocity $v_{\mathrm{c}} \sim W_{12} / \eta$ is reached, "forced wetting" is observed, where the lubricant enters the interface between polymer and steel, the adhesive contact is lost and the system enters the mixed lubrication regime.

The experiments described in the current paper focus on a study of the COF for different velocity and lubrication regimes. From an applied perspective, it would be desirable to be able to draw conclusions from this type of measurement on the wear behavior of lubricated thermoplastic polymers. The wear of polymers can be approached on a fundamental level as the interaction of cohesive and interfacial wear [16]. The abrasive effect of the frictional partner of the polymer needs to be described on the one hand by the mechanical characteristics of the polymer and on the other hand by the surface structure of the frictional partner and the occurring local pressure, resulting in different "modes of interaction", i.e., smoothing, ploughing or cutting [17-19]. Ultimately, the tribological interactions need to be viewed as a highly dynamic process, where, e.g., local heating of the polymer by the frictional power changes its mechanical characteristics (up to a local melting) and the transfer of the polymer to the frictional partner changes the structure and property of the contact partner. Only few research works focus on the influence of lubricants on the above-mentioned effects. These publications describe the influence of chemistry [20], partial wetting [21,22] and the applicability of different experimental methods in order to study the phenomena [23,24].

Despite the complexity of the tribology of lubricated polymers, the adhesive interaction between polymer and frictional partner generally occurs either by the contact of the polymer with a "smooth" frictional partner, or by the local contact of the polymer with the asperities of a "rough" frictional partner. As a result, studies of the adhesive friction of lubricated polymers with "smooth" surfaces can potentially yield some insight into some aspects of the friction of lubricated polymers with rough surfaces.

\section{Materials and Methods}

If not stated otherwise, all data refer to properties at $25^{\circ} \mathrm{C}$ and experiments were done at $25^{\circ} \mathrm{C}$.

\subsection{Materials}

Tribological experiments were conducted on a lubricated poly(ether ether ketone) (PEEK)or polyamide (PA46)-steel tribosystem. Unfilled PEEK450sf (PEEK) was obtained from Ensinger $\mathrm{GmbH}$ (Nufringen, Germany) and unfilled Stanyl (PA46) was obtained from Schmidt + Bartl GmbH (Villingen-Schwenningen, Germany). In addition, unfilled PEEK KetaSpire ${ }^{\circledR}$ KT-880 NT from Albis Plastic GmbH (Hamburg, Germany) was used for some experiments. A 100Cr6 steel ball that was integrated in the tribometer was used as tribological partner. Pentaerythrite ester (PEEs), trimellitic acid ester (TAEs) and synthetic base oil (PAO, viscosity $\eta=117 \mathrm{mPa} \cdot \mathrm{s}$ at $25^{\circ} \mathrm{C}$ ) were obtained from Klüber Lubrication München SE \& Co. KG (München, Germany). Furthermore, Ethylene Glycole (Fluka, München, Germany), Glycerine (Carl Roth GmbH + Co KG, Karlsruhe, Germany), and Poly(1-decene) (VWR International $\mathrm{GmbH}$, Bruchsal, Germany) were purchased and used as lubricating media. By doing so, two groups of liquids with comparable viscosity were obtained. One group is representing industrial base oils and the other group widely used obtainable chemicals covering a broad spectrum of cohesive energies being nonpolar or highly polar. The PEEK and PA46 samples were prepared in the form of rectangular plates $(6 \times 3 \times 15 \mathrm{~mm})$ which were cut from tapered tensile test specimens (PEEK) or from molding plates (PA46). 


\subsection{Tribological Experiments}

Ball-on-plate experiments were used to record a Stribeck curve and carry out oscillatory tribological experiments. A rotational rheometer developed by Anton Paar $\mathrm{GmbH}$ was used which was equipped with a tribological measuring unit. In this way, very low velocities and deflections $\left(1 \times 10^{-4} \mathrm{~mm} / \mathrm{s}\right)$ can be obtained via a rotation of polished steel ball. To achieve stable and reproducible system conditions a testing sequence was applied to form a run-in situation [5]. Conducting the oscillatory experiments in this range of sliding speeds (oscillatory mode) makes it possible to record friction data in the static friction and boundary-lubrication regimes. In the continuous gliding mode (Stribeck mode), conventional unidirectional rotational experiments are conducted with steadily increasing gliding speeds ranging from $1 \times 10^{-4} \mathrm{~mm} / \mathrm{s}$ to $1.4 \times 10^{3} \mathrm{~mm} / \mathrm{s}$. Stribeck mode experiments collect friction data in the boundary-lubrication and mixed-lubrication friction regimes. For a detailed description of the experimental set-up, we refer to the previous study [5].

\subsection{Contact Angle Measurements}

The sessile drop method of $2 \mu \mathrm{L}$ droplets on the samples was used on a contact angle measurement device produced by Data Physics GmbH. Three standard liquids (water [25], ethylene glycol [25] and diiodomethane [26]) were used to characterize the unknown surface and interfacial energies. The droplet profile is recognized and recorded by the instrument's software and the contact angle is calculated automatically. All the calculations and interpolations were carried out according to the Equations (3)-(8). Again, a detailed description of the procedure was given in the previous study [5].

\section{Results}

\subsection{Contact Angle Measurements and Interaction Energies}

All the calculations and interpolations were carried out according to the procedure described by Wahed et al. [5] and the resulting energies were calculated (Table 1). The robustness of the standard regression method of surface and cohesive energies was increased by including float glass and Mg-PSZ (magnesia partially stabilized zirconia) ceramic surfaces as additional materials and fitting all parameters in all equations in one attempt.

Table 1. Table of lubricants.

\begin{tabular}{|c|c|c|c|c|c|c|c|}
\hline \multirow[t]{2}{*}{ Lubricant } & \multirow{2}{*}{$\begin{array}{c}\text { Viscosity } \\
\text { At } 25^{\circ} \mathrm{C} \\
{[\mathrm{mPa} \cdot \mathrm{s}]}\end{array}$} & \multirow[t]{2}{*}{$\begin{array}{c}\text { Contact Angle } \\
\text { on Steel }\left[{ }^{\circ}\right]\end{array}$} & \multicolumn{3}{|c|}{ PA46 } & \multicolumn{2}{|c|}{ PEEK 450sf } \\
\hline & & & $\begin{array}{l}W_{\text {solving }} \\
{[\mathrm{mN} / \mathrm{m}]}\end{array}$ & $\begin{array}{c}W_{\text {spreading }} \\
{[\mathrm{mN} / \mathrm{m}]}\end{array}$ & $W_{\text {solving }} / \gamma_{13}$ & $\begin{array}{l}W_{\text {solving }} \\
{[\mathrm{mN} / \mathrm{m}]}\end{array}$ & $\begin{array}{c}W_{\text {spreading }} \\
{[\mathrm{mN} / \mathrm{m}]}\end{array}$ \\
\hline pentaerythrite ester (PEEs) & 109 & 23.4 & 4.6 & 1.2 & 1.0 & 2.5 & -1.3 \\
\hline trimellitic acid ester (TAEs) & 153 & 23.9 & 4.8 & 2.1 & 1.0 & 2.6 & -1.8 \\
\hline ethylen glycol & 21 & 54.2 & 3.5 & 4.0 & 1.0 & 16.3 & 15.8 \\
\hline water & 1 & 78.7 & -22.8 & 27.2 & 1.3 & 39.7 & 51.9 \\
\hline glycerine & 1420 & 64.5 & 5.4 & 11.5 & 1.0 & 7.5 & 8.0 \\
\hline $30 \%$ water, $70 \%$ glycerine & 20 & 72.2 & -0.9 & 11.6 & 1.1 & 12.2 & 13.5 \\
\hline poly(1-decene) & 25 & 16.7 & 3.9 & 2.3 & 1.0 & 3.9 & -0.6 \\
\hline
\end{tabular}

\subsection{Static Friction and the Onset of Gliding}

An essential question for the study of the transition from static to dynamic friction is the detection of the onset of gliding. A continuous experiment shows an unstable friction behavior when gliding is initiated. By using an oscillatory deflection method, the transition can be monitored by a sequence of transition events and one can distinguish between the elastic and viscous response of the system. The evolution of the phase shift between deflection and gliding response can be estimated. For polymer-lubricant systems with an interaction parameter $W_{\text {solving }} / \gamma_{13}$ close to unity, typically a well-defined transition between static and dynamic friction is observed. Systems with an interaction parameter larger than unity (e.g., PA46 and water) show a complex and more gradual transition from 
sticking to gliding, which is indicative for the interaction of the lubricant and the polymer surface. For these cases, the definition of an adhesion threshold is, for some experiments, somewhat ambiguous. Using a data acquisition rate of 10 data points per decade the loading torque was increased on a logarithmic scale and the oscillation frequency was $1 \mathrm{~Hz}$.

For PEEK [6] and PA46, the two ester oils TAEs and PEEs were tested in comparison with the non-lubricated, "dry" system. For PEEK [5] the previous study showed that the oscillations with amplitude less than $1 \mu \mathrm{m}$ at a frequency of $1 \mathrm{~Hz}$ show an elastic behavior. This is also observed for PA46. All measurements showed an increase of the loss factor tan $\delta$ to values above one, when the amplitude was increased further after a maximum value of the tangential force was reached. This indicates that either the thermoplastic polymer or the contact starts exhibiting a viscoelastic behavior. In the previous paper [5], it was already concluded that this marks the onset of gliding, which is the beginning of the "state behavior" of the rate-and-state model for the dry and lubricated PEEK-steel tribosystem.

The PEEK-steel and PA46-steel tribosystems show different spreading tendencies for the two ester-based oils PEEs and TAEs. Whereas the ester oils are spreading lubricants, for the PEEK-steel combination, the same lubricants do not spread readily into the PA46-steel tribological contact. When comparing the ratio of friction force and normal force $\left(\mathrm{F}_{\mathrm{r}} / \mathrm{F}_{\mathrm{n}}\right)$ at the onset of gliding for the dry and lubricated tribosystem, we observe that the lubricants reduce the transversal force where the onset of gliding is observed with respect to the dry contact when they are able to spread into the tribological contact. In the non-spreading PA46-steel system, the same lubricants do not result in a lower $F_{r} / F_{n}$ ratio at the onset of gliding; in fact, we observe slightly higher $F_{r} / F_{n}$ ratios for the lubricated system (Figure 1). The adhesive contact of the non-spreading system also results in an elongated stiction phase exhibiting a gradual transition from static to dynamic friction over several decades of the displacement rate $\mathrm{s} \cdot \mathrm{f}$ (s: amplitude of ball surface displacement, $\mathrm{f}$ : frequency of oscillation was $1 \mathrm{~Hz}$ ) (see Figure 1 , right hand side).
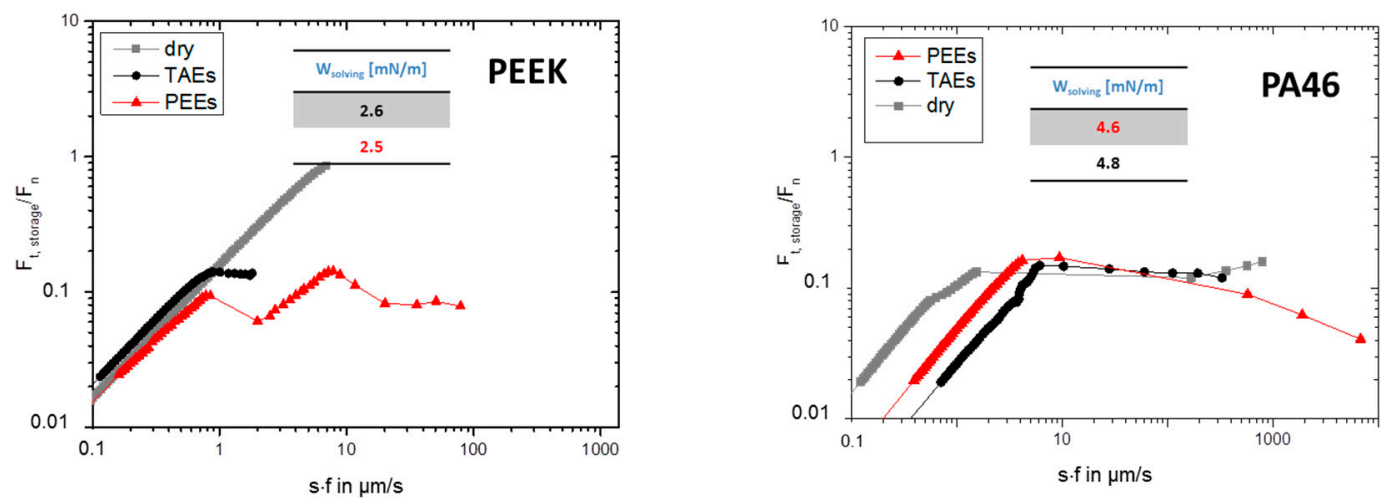

Figure 1. The storage part of the ratio of tangential and normal loading or coefficient of friction vs. sliding of PEEK and PA46 against steel 100Cr6 in dry (grey) and lubricated condition with PEEs (red, pentaerythrite ester) and TAEs (black, trimellitic acid ester). The experiments were conducted in the oscillatory mode under a normal force of $9 \mathrm{~N}$ resulting in an elastic spherical contact with Hertzian pressure of $47 \mathrm{MPa}$ and a temperature of $25^{\circ} \mathrm{C}$.

When the adhesive contact is not lost by a lubricant film which enters the interface between polymer and tribological partner, the lubricant might nevertheless alter the characteristics of the surface layer of the polymer when it is able to migrate into the surface and mobilizes the polymer chains. In the earlier work [5] we suggested that $W_{\text {solving }}$ is an indication for the tendency of a lubricant to mobilize the polymer chains close to the surface of the tribological contact. 
Figure 2 shows the transition from static friction to gliding for the PA46-steel tribosystem under not-lubricated (dry) conditions and lubricated with five different non-spreading lubricants. For all cases we can therefore assume an intact adhesive contact between PA46 and the steel frictional partner at low displacement rates. A monotonous increase of the ratio $F_{r} / F_{n}$ up to a maximum value is observed which corresponds to the adhesion threshold. The differences in the friction behavior, represented by $F_{r} / F_{n}$, correlate with the value of $W_{\text {solving }}$ which is obtained for the combination of PA46 and the lubricant. Among the lubricated systems, the highest positive $W_{\text {solving }}$ value is assigned to the glycerine-PA46 system, which features the highest adhesion threshold prior to sliding. The lowest negative $W_{\text {solving }}$ value is assigned to the water curve, which shows the most steadily increasing static friction and the lowest adhesion threshold prior to sliding. For the other lubricants, the ranking of the adhesion thresholds correlates well with the magnitude of $W_{\text {solving. }}$ The $\mathrm{F}_{\mathrm{r}} / \mathrm{F}_{\mathrm{n}} \mathrm{vs}$. s.f curves of systems which show an increased interaction parameter (i.e., PA46/water and PA46/water-glycerine) exhibit in the double-logarithmic representation a cross-over to a line with a smaller slope.

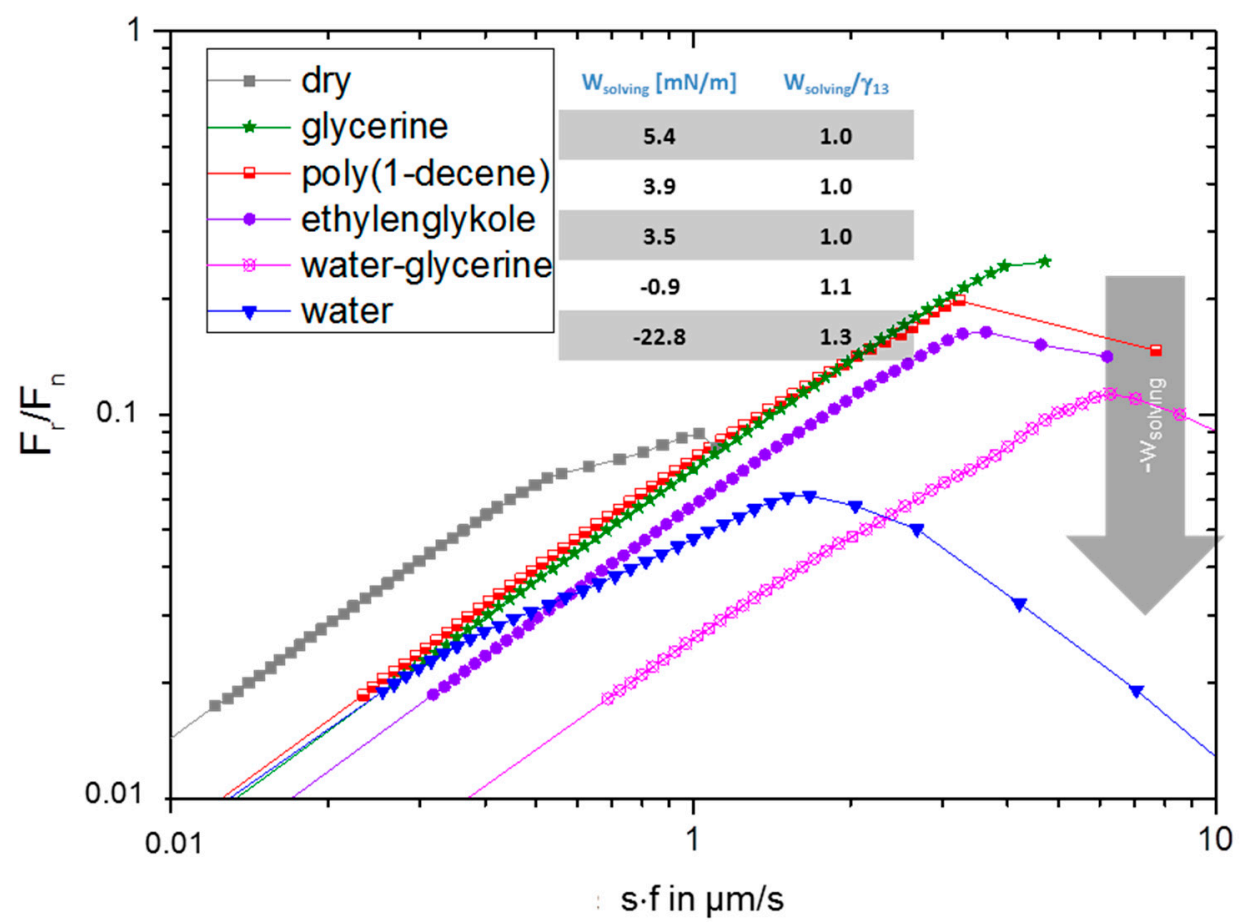

Figure 2. The ratio of tangential and normal loading or coefficient of friction vs. sliding of PA46 against steel 100Cr6 in dry (grey) and lubricated conditions. The experiments were conducted in the oscillatory mode under a normal force of $9 \mathrm{~N}$ resulting in an elastic spherical contact with Hertzian pressure of $47 \mathrm{MPa}$ and a temperature of $25^{\circ} \mathrm{C}$.

\subsection{Dry Boundary Friction, Forced Wetting, Lubricated Boundary Friction and Mixed Lubrication}

In contrast to the oscillatory mode experiments, the Stribeck experiments are true gliding tests. In these tests, the development of the COF with increasing sliding velocity is recorded. The velocities for which the COF was recorded were chosen at equidistant intervals on a logarithmic velocity scale. In order to determine the $\mathrm{COF}$, the velocity was kept constant for one second during which the data were acquired. 
In the previous work [5], PEEK lubricated with PEEs and TAEs was studied. The spreading energy $W_{\text {spreading }}$ is negative for these lubricants, i.e., the system gains energy when the lubricants spread into the tribological contact. When comparing the lubricated systems with the non-lubricated, dry system, the lubricants result in a significant decrease of the COF. The lubricant with the higher tendency to invade the interface between PEEK and steel showed the lower dynamic friction (see Figure 3). Due to the tendency of the oils to spread spontaneously into the PEEK-steel interface, a smooth transition from the adhesion threshold to gliding was recorded.

PA46, steel and the lubricants PEEs and TAEs are non-spreading systems, i.e., the lubricants do not spread readily into the tribological contact. When analyzing the tribological behavior of these systems, no reduction of the COF with respect to the "dry" system is observed for slow sliding velocities in the range of $0.1-5 \mathrm{~mm} / \mathrm{s}$; in fact, for velocities up to $1 \mathrm{~mm} / \mathrm{s}$ we observe a slight increase of the COF for the lubricated systems. As for the spreading systems, the ranking of the COF for the non-spreading lubricated systems corresponds to the order of $W_{\text {spreading, }}$, i.e., the lubricant with the lower value of $W_{\text {spreading }}$ shows the lower COF. A comparison with the PEEK-steel tribosystem shows that the reversal of the order of $W_{\text {spreading }}$ of the lubricants for the PA46-steel tribosystem results in a reversal of the order of the COF for these lubricants. Since PEEs and TAEs have similar viscosities, differences in $W_{\text {spreading }}$ strongly influence the differences in the COF of these systems.
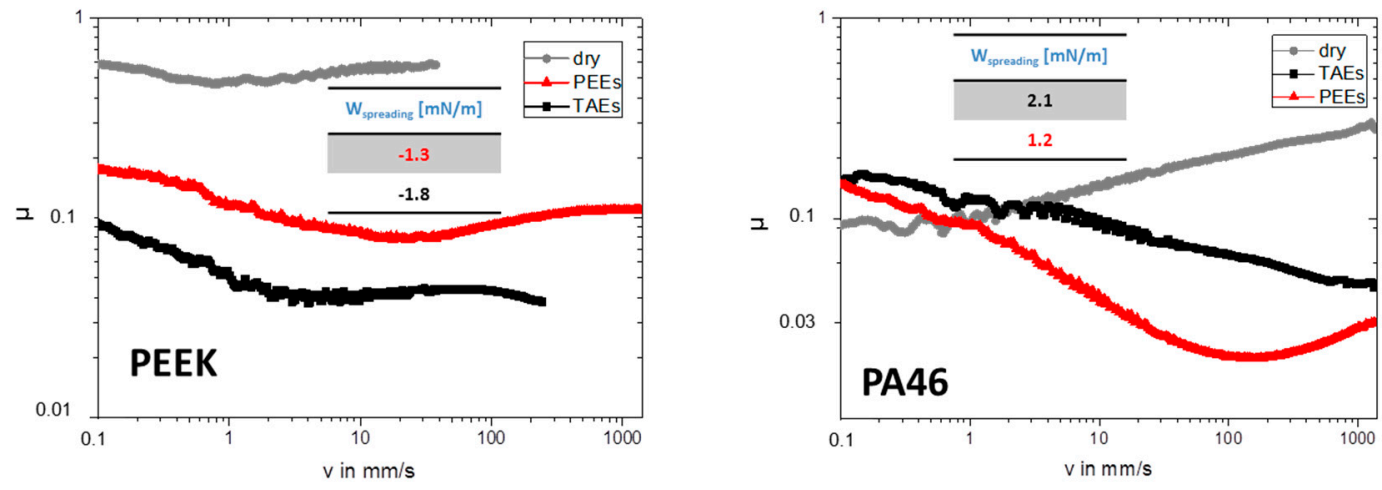

Figure 3. Friction coefficient vs. sliding speed for PEEK and PA46 against steel 100Cr6 in dry (grey) and a lubricated condition with PEEs (red, pentaerythrite ester) and TAEs (black, trimellitic acid ester). The experiments were conducted in the Stribeck mode under a normal force of $9 \mathrm{~N}$ resulting in an elastic spherical contact with Hertzian pressure of $47 \mathrm{MPa}$ and temperature of $25^{\circ} \mathrm{C}$.

The PEEK systems studied in the previous work [5] exhibited a steady decrease of the COF until a minimum value of the COF was reached at approximately $1 \mathrm{~mm} / \mathrm{s}$ for the dry system and $3 \mathrm{~mm} / \mathrm{s}$ (TAEs) or $30 \mathrm{~mm} / \mathrm{s}$ (PEEs) for the lubricated systems. Considering the low velocities, the applied pressure (47 MPa) and the viscosity of the lubricants, we concluded that it is unlikely that the minima of the COF mark the transition from mixed to hydrodynamic lubrication. The initial decrease of the COF was associated with an "aging" of the state of the PEEK-steel interface as it is described in abstract terms by the rate-and-state models. For the PA46-steel interface, the development of the COF does not show features which allow a distinction of the "state" behavior and the behavior in the mixed lubrication regime.

The observation that the use of non-spreading lubricants resulted for low sliding velocities in a higher COF compared with the "dry" system and that the order of the COF corresponds to the value of $W_{\text {spreading }}$ can be confirmed for the PEEK-steel and PA46-steel combination if the lubricants ethylene glycol and glycerine are used (see Figure 4). Both lubricants show a markedly different tendency to spread (the difference in spreading energy for these lubricants amounts to one-quarter of the cohesion energy of the lubricants), and both lubricants result in non-spreading systems for the PEEK-steel and PA46-steel combinations. Due to the high dynamic viscosity of glycerine, the onset of the hydrodynamic lubrication regime lies for both polymers within the range of sliding velocities 
which were studied in the experiments. The onset of the hydrodynamic regime of the PEEK-steel system occurs at a slower sliding speed than for the PA46-steel system $(v=10 \mathrm{~mm} / \mathrm{s}$ for PEEK-steel, $v=100 \mathrm{~mm} / \mathrm{s}$ for PA46-steel). The higher tendency of glycerine to mobilize the polymer chains at the PA46 surface (PEEK: $W_{\text {solving }}=7.5 \mathrm{mN} / \mathrm{m} ;$ PA46: $W_{\text {solving }}=5.4 \mathrm{mN} / \mathrm{m}$ ) can potentially explain the shift of the onset of the hydrodynamic regime. A softer surface area with more mobile polymer chains can possibly account for the delayed total separation of the friction partners by the formation of a lubricant film.
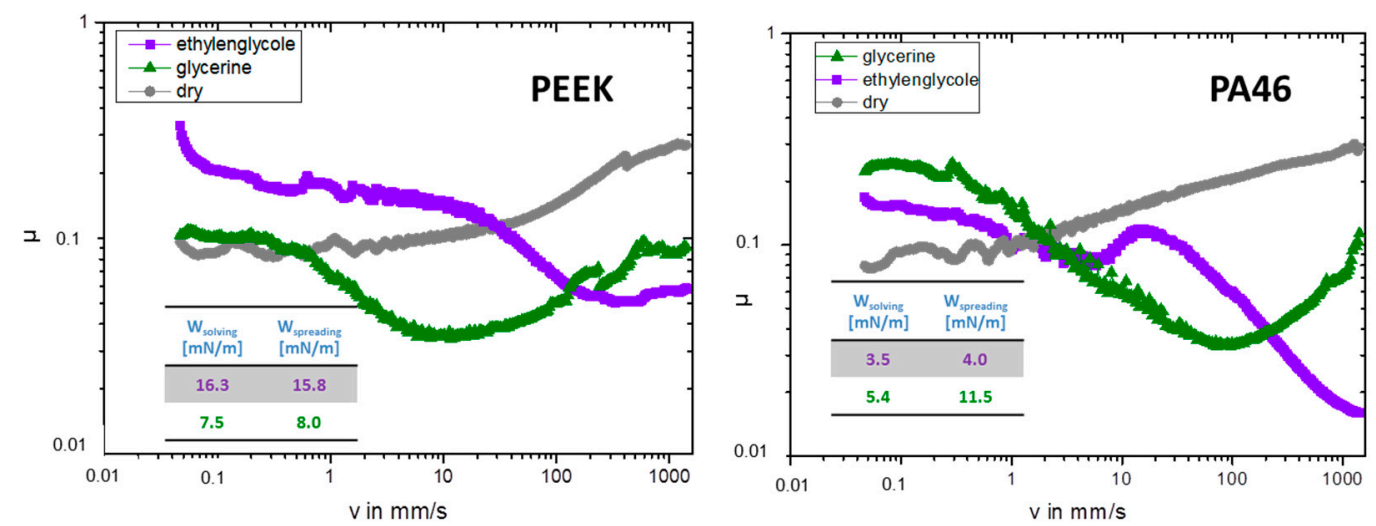

Figure 4. Friction coefficient vs. sliding speed for PEEK and PA46 against steel 100Cr6 dry (grey) and lubricated. The experiments were conducted in the Stribeck mode at $25^{\circ} \mathrm{C}$ applying a normal force of $9 \mathrm{~N}$ which results in an elastic spherical contact with a Hertzian pressure of $47 \mathrm{MPa}$.

By mixing the lubricants water and glycerine in different ratios, it is possible to prepare lubricants with viscosities which can vary by three orders of magnitude. In order to investigate the influence of the viscosity on the onset of the hydrodynamic regime, the PA46-steel system was studied with three lubricants: water $(\eta=1 \mathrm{mPa} \cdot \mathrm{s})$, a $30 \% / 70 \%$ water $/$ glycerine mixture $(\eta=20 \mathrm{mPa} \cdot \mathrm{s})$, and glycerine $(\eta=1420 \mathrm{mPa} \cdot \mathrm{s})$. PA46 and steel form with all three lubricants non-spreading systems. Since the polar component of the cohesive energy is different for the three lubricants, the resulting surface and interfacial energies vary, which should be taken into account when analyzing the tribological experiments. Both oscillatory tribological and Stribeck-type gliding experiments were carried out on the three systems. The oscillatory tribological experiments showed that the transition from static to dynamic friction occurred at displacement rates s.f ranging from $1 \mathrm{~mm} / \mathrm{s}$ to $10 \mathrm{~mm} / \mathrm{s}$. The height of the adhesion threshold correlates well with the magnitude of $W_{\text {solving. }}$. When assigning a sliding velocity of $1 \mu \mathrm{m} / \mathrm{s}$ to oscillating amplitudes of $1 \mu \mathrm{m}$ at $1 \mathrm{~Hz}$ in the oscillatory experiments, both types of experiments can be displayed in a single graph-with the sliding velocity as $\mathrm{x}$-coordinate and the ratio $F_{r} / F_{n}$ or the COF $\mu$ as $y$-coordinate. The results for the lubricated PA46-steel systems displayed in Figure 5 show that the values $F_{r} / F_{n}$ at the onset of gliding which were obtained from of the oscillatory measurements match the values of the COF at very low sliding velocities which were obtained from the Stribeck-type of measurements. As a consequence, the curves obtained from the two types of experiments can be formally joined in a single graph for the studied tribosystems. The velocity where forced wetting of the tribological contact occurs is marked by a sharp decline of the COF. The sharp decline of the COF is followed by a minimum of the COF which corresponds to the onset of the hydrodynamic friction which can be identified for the two lubricants with the higher viscosities. The onset of the forced lubrication agrees very well with the theoretical $1 / \eta$ dependency. 


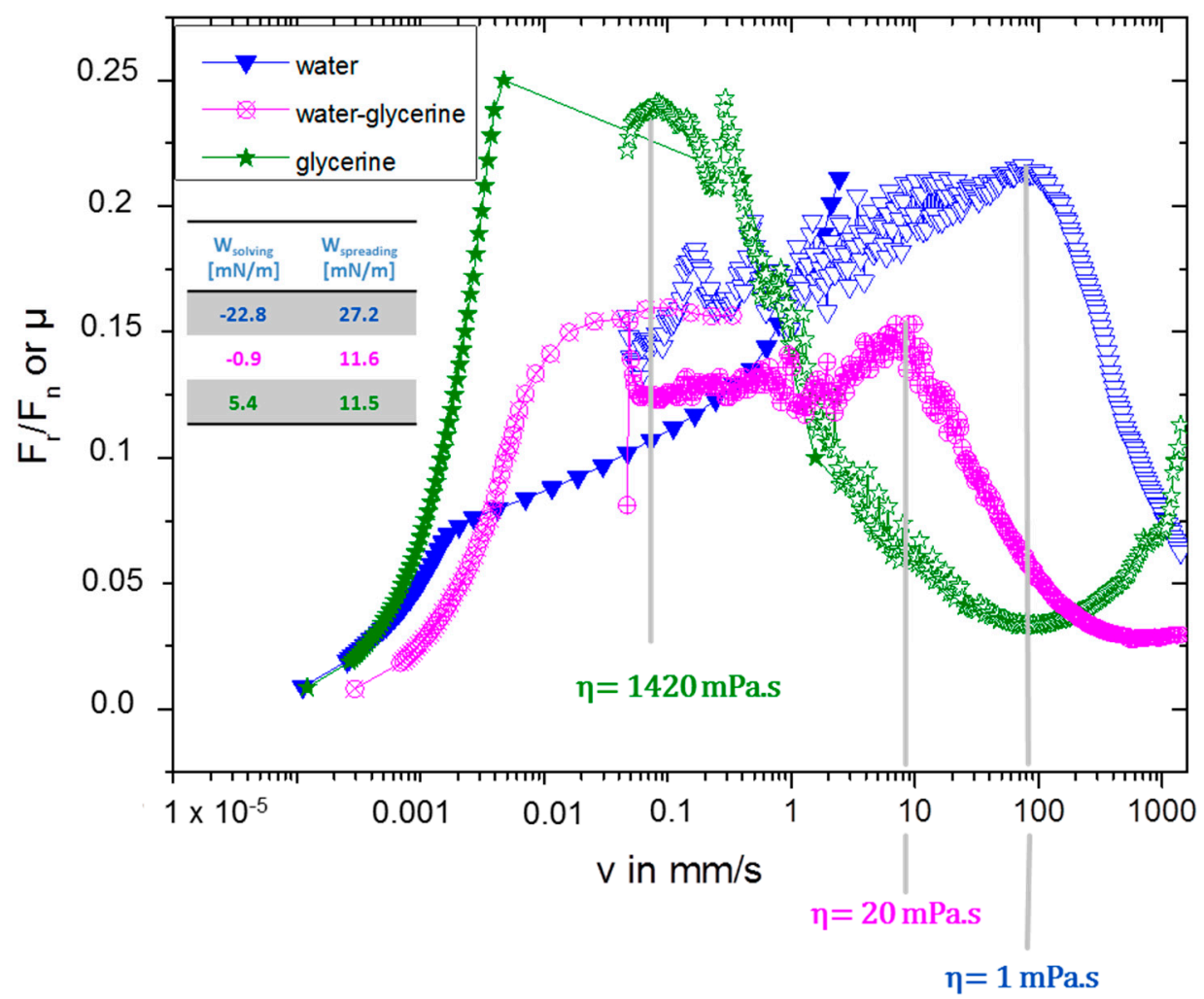

Figure 5. The ratio of tangential and normal loading or coefficient of friction vs. the sliding velocity for PA46 against steel 100Cr6 lubricated with water, glycerine and a 30\%/70\% water/glycerine mixture. The experiments were conducted in the oscillatory mode and in the Stribeck-mode at $25{ }^{\circ} \mathrm{C}$ applying a normal force of $9 \mathrm{~N}$ resulting in an elastic spherical contact with Hertzian pressure of $47 \mathrm{MPa}$.

In addition, the $1 / \eta$ dependence of the velocity which marks the onset of the wetting of the tribological contact was tested for the PEEK-steel tribological contact lubricated with synthetic base oil (PAO). In these experiments, the viscosity of the lubricant was varied by increasing the temperature of the system, thus, decreasing the viscosity of the oil. Experiments were carried out at $25^{\circ} \mathrm{C}$ and $175^{\circ} \mathrm{C}$, for two different normal forces $(\mathrm{F}=3 \mathrm{~N}$ and $\mathrm{F}=9 \mathrm{~N})$. The viscosity of the lubricant was determined from data obtained from the manufacturer. Again, the $1 / \eta$ dependence of the velocity that marks the onset of the wetting of the contact was confirmed, see Figure 6. The temperature and pressure variation have no significant influence on the COF at low sliding velocities: at $v=0.1 \mathrm{~mm} / \mathrm{s}$, a COF slightly above 0.1 is observed.

This observation is in contrast to results for the non-lubricated PEEK-steel system: Figure 7 shows the results of oscillatory tribology and Stribeck-mode experiments at different normal forces and temperatures. The results of the two types of experiments were combined in one graph similar to the approach taken in Figure 5. When increasing the normal force at $T=25^{\circ} \mathrm{C}$, a decrease in the COF was observed as it is expected for the adhesive contact of a low-modulus frictional partner: the "real" contact area does not increase proportional to the increasing normal force for polymeric materials. When increasing the temperature from $T=25^{\circ} \mathrm{C}$ to $T=175^{\circ} \mathrm{C}$ one would in general expect an increase of the real contact area due to the softening of the polymer and, thus, a higher value of the COF. The experimental results show the opposite: the COF decreases with increasing temperature. This behavior can be explained with the rate-and-state model which assumes a thermally activated rate process for the slipping motion. The lowering of the $\mathrm{COF}$ for higher temperatures agrees well with the increased thermal activation of the slipping process which reduces the life-time of the "bonded" state. This clearly visible systematic change could not be found in the experiments with a lubricant which spreads into the tribological contact (see Figure 6). 


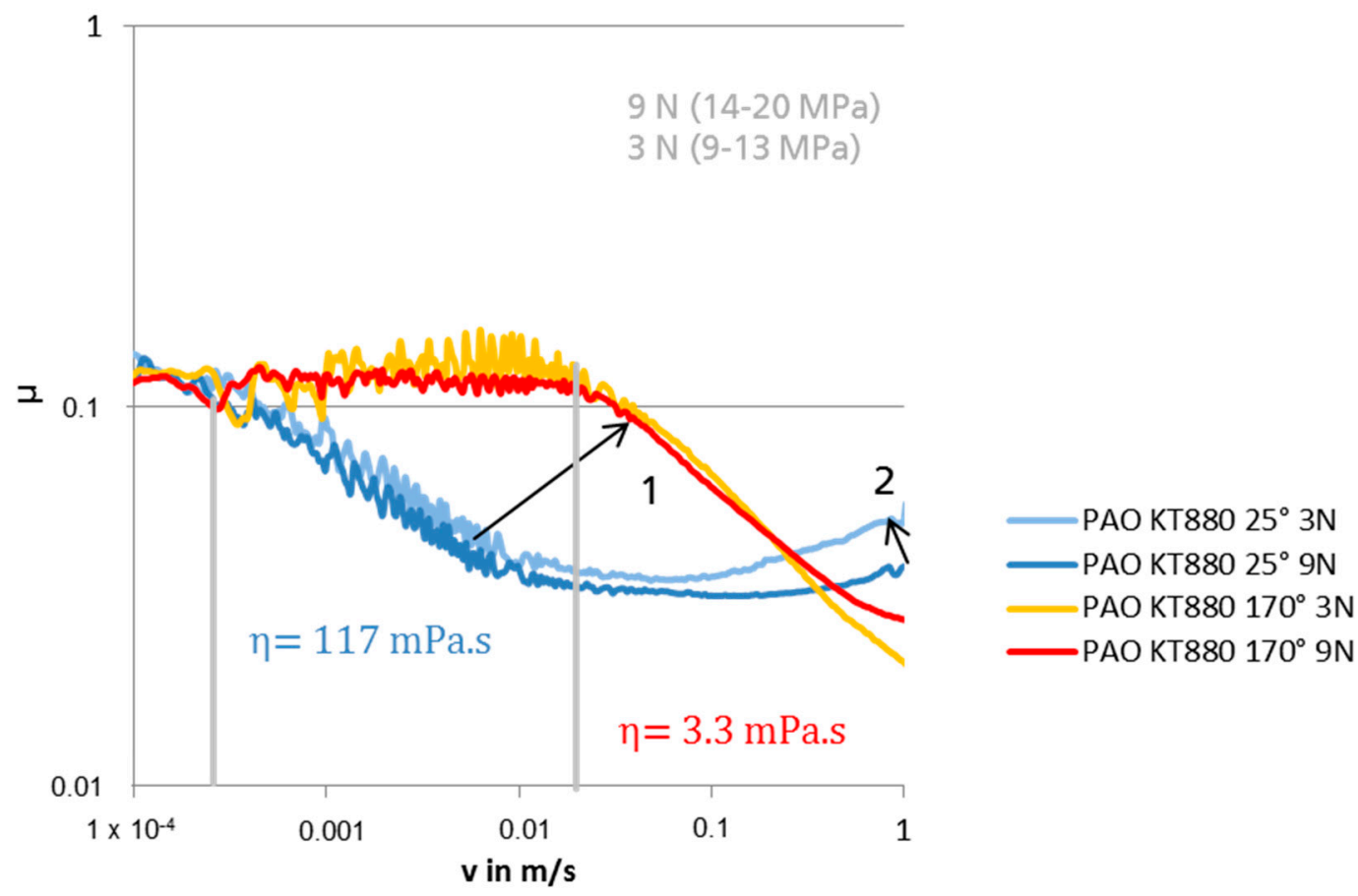

Figure 6. Friction coefficient vs. sliding speed for PEEK (KT880 in this case) against steel 100Cr6 lubricated with PAO (synthetic base oil) $T=25^{\circ} \mathrm{C}$ and $T=175^{\circ} \mathrm{C}$. The experiments were conducted in the Stribeck-mode applying a normal force of $3 \mathrm{~N}$ and $9 \mathrm{~N}$ which results in an elastic spherical contact with Hertzian pressure of $33 \mathrm{MPa} 47 \mathrm{MPa}$.
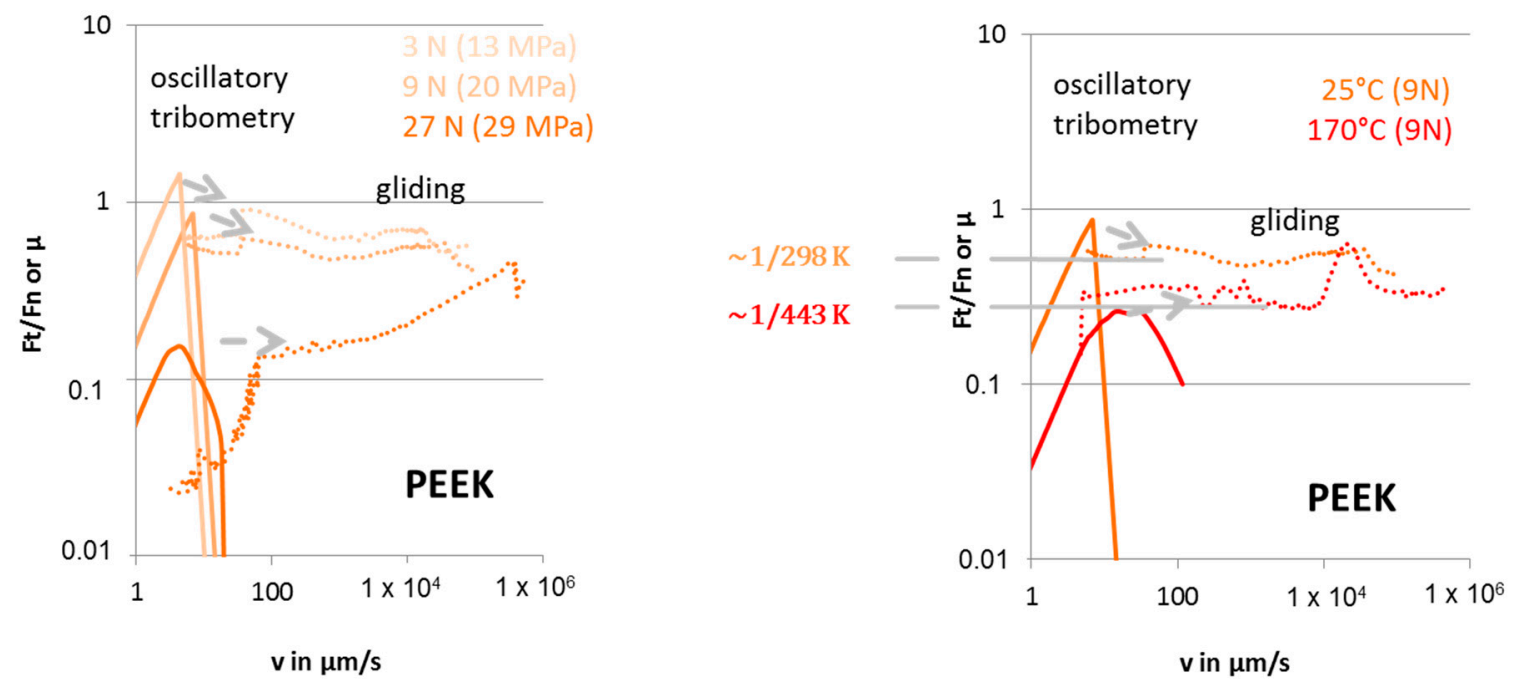

Figure 7. The ratio of tangential and normal loading or coefficient of friction and the friction coefficient vs. sliding of PEEK against steel 100Cr6 without lubrication. The experiments were conducted in the oscillatory mode and in the Stribeck mode at $25^{\circ} \mathrm{C}$ (left hand side) applying a normal force of $3 \mathrm{~N}$, $9 \mathrm{~N}$ and $27 \mathrm{~N}$ and at $25^{\circ} \mathrm{C}$ and $170{ }^{\circ} \mathrm{C}$ applying a normal force of $9 \mathrm{~N}$ (right hand side).

\section{Discussion}

\subsection{Static Friction and the Onset of Gliding}

In the previous paper [6] we concluded that higher values for the work of solving result in higher values for the static friction's adhesion threshold for moderately spreading lubricants. In the current work we focus on non-spreading systems. When using the same oils (TAEs and PEEs) as lubricants for PA46 we obtain a non-spreading system with a similar ranking of $W_{\text {solving }}$ : in both cases $W_{\text {solving }}$ (TAEs) 
is approximately $5 \%$ larger than $W_{\text {solving }}$ (PEEs). The results for PA46 hint that we observe the opposite tendency for non-spreading systems: the adhesion threshold decreases with increasing $W_{\text {solving }}$. When non-spreading systems with different levels of the spreading energy $W_{\text {spreading }}>0$ were studied, this finding was confirmed: a higher solving tendency (i.e., lower values for $W_{\text {solving }}$ ) and an increased interaction parameter typically resulted in a reduced friction and elasticity. A plasticizing or softening of the surface of the thermoplastic polymer can possibly explain the trends in the tribological behavior observed for lubricants with higher solving tendencies: a "softer" surface will result in less resistance if asperities of the steel surface are moved through the polymer surface. This effect should be quite pronounced for water-based lubricants since it is well known that water acts as a plasticizer for PA46 and can be sorbed by the polymer bulk.

\subsection{Dry Boundary Friction, Forced Wetting, Lubricated Boundary Friction and Mixed Lubrication}

The finding of the previous study that the tendency of a lubricant to spread into the tribological contact is indicative for the COF at low sliding velocities (the "rate-and-state" part of the Stribeck curve of lubricated PEEK) could be confirmed for non-spreading systems. In particular it was shown that a reversal of the ranking of $W_{\text {spreading }}$ when switching from PEEK to PA46 as frictional partner resulted in a reversal of the order of the $\mathrm{COF}$ at low sliding velocities. This indicates that the lubricant also influences the non-spreading contact. A possible explanation for this finding could be the sorption of the lubricant to the thermoplastic body and a prolonged stiction phase, or a partial spreading or a forced-wetting cone at the front of the interface and increased friction. In contrast to the lubricated PEEK-steel system with a transient wetting transition, we did not observe a "state-behavior" (i.e., an initial decrease of the COF at low velocities) of the non-spreading systems. The rate-and-state behavior that should be the origin of a friction minimum might be hidden by the transition to forced wetting.

The dependence of the critical velocity for forced wetting or for squeeze out $v \sim 1 / \eta \quad[21,22]$ was confirmed at room temperature for PA46 using different viscosities of the water-glycerine system. It was also confirmed for PAO as lubricant for PEEK but when varying the viscosity by temperature variation.

The fundamental aspect of the Arrhenius-activated slip (see Equation (1)) [6] could be verified for dry boundary friction of PEEK by a significant increase in temperature. The friction is reduced for higher temperatures when a thermally increased debonding rate reduces the fraction of polymer chains bonded to the frictional partner.

\section{Conclusions}

Spontaneous spreading of the lubricant into the contact surface between the tribopartners is expected when the overall transition will form exotherm energy. Therefore, negative values for work-of-spreading $W_{\text {spreading }}$ are expected for spreading systems. This is the case for PEEK and steel: the wetting of the two frictional partners with industrial oil does not need to be forced by, e.g., applying a suitable sliding speed. A positive value for work-of-spreading $W_{\text {spreading }}$ indicates that-from a purely energetic point of view-lubricants will not move into the interface between the frictional partners or, as in the case of the chosen tribological experiments with interference below $100 \%$, will move out the interface. The non-spreading systems typically show an increased COF. Following Schallamach's approach of mobile polymer chains, which temporarily bond to the frictional partner, polymers can form adhesive junctions to steel and bridge the gap which is formed by the lubricant of a spreading system. In the case of a non-spreading contact, the lubricant could (a) be sorbed into the bulk of the polymer to enter the interface or (b) eventually form an elastic squeeze-out cone at the front side of the gliding interface. All cases will be followed by a change of overall adhesive bonding. The friction interface for spreading or non-spreading contact could undergo a dynamic rupture process from stiction to gliding. This description is often based on local rate-and-state conditions and locally distributed instabilities. Rupture is followed by slipping events with partial intake zones of a lubricant film that add to or subtract from rupture energy. 
The concept of the solving tendency of lubricants for thermoplastic polymers was applied for a wider range of lubricants and polymers, and refined for the static friction and the onset of gliding. The sorption of a lubricant into a thermoplastic polymer can increase mobility of the polymer surface, decrease viscoelastic loss and lower the friction by microploughing at rough contact with asperities.

The tendency of a lubricant to spread into the tribological contact determines the reduced friction in the rate-and-state case for lubricating oils, especially for boundary friction, but also for forced wetting and mixed lubrication. The onset of forced wetting depends mainly on the viscosity of the lubricant. The critical velocity for different lubricants is inverse proportional to their viscosity.

The understanding of transitions in the frictional behavior of lubricated contacts (e.g., the transition from stiction to gliding or the onset of forced wetting) is of utmost importance for many applications. Although the macroscopic methods applied in this work do not provide a detailed insight into microscopic mechanisms, their results are in agreement with the corresponding models. The fundamental benefit of the method discussed in this paper is the fairly basic and robust approach to screen systems over a wide range of conditions. From an applied perspective, it is attractive to link state-of-the-art interface theories to experimental approaches which are easy to perform and can find wide-spread use in industry. Possible applications are in drivetrain technology, for bearings and seals.

Author Contributions: Conceptualization, C.K.; Investigation, S.A.A.-W.; Writing-review \& editing, R.J. and M.S.

Funding: The work and the research of Sherif Ahmed Abdel-Wahed were funded by the German Academic Exchange Service (DAAD) for the GERLS scholarship (programme No. 57147166, ID No. 91567277).

Conflicts of Interest: The authors declare no conflict of interest.

\section{References}

1. Zhang, S.W. State-of-the-art of polymer tribology. Tribol. Int. 1998, 31, 49-60. [CrossRef]

2. Scherge, M.; Kramlich, J.; Böttcher, R.; Hoppe, T. Running-in due to material transfer of lubricated steel/PA46 (aliphatic polyamide) contacts. Wear 2013, 301, 758-762. [CrossRef]

3. Samyn, P.; Schoukens, G. Calculation and Significance of the Maximum Polymer Surface Temperature $\mathrm{T}$ * in Reciprocating Cylinder-On-Plate Sliding. Polym. Eng. Sci. 2008, 48, 774-785. [CrossRef]

4. Campen, S.; Green, J.; Lamb, G.; Atkinson, D.; Spikes, H. On the Increase in Boundary Friction with Sliding Speed. Tribol. Lett. 2012, 48, 237-248. [CrossRef]

5. Abdel-Wahed, S.A.; Koplin, C.; Jaeger, R.; Scherge, M. On the Transition from Static to Dynamic Boundary Friction of Lubricated PEEK for a Spreading Adhesive Contact by Macroscopic Oscillatory Tribometry. Lubricants 2017, 5, 21. [CrossRef]

6. Schallamach, A. A theory of dynamic rubber friction. Wear 1963, 6, 375-382. [CrossRef]

7. Chaudhury, M.K. Rate-Dependent Fracture at Adhesive Interface. J. Phys. Chem. B 1999, 103, $6562-6566$. [CrossRef]

8. Vorvolakos, K.; Chaudhury, M.K. The Effects of Molecular Weight and Temperature on the Kinetic Friction of Silicone Rubbers. Langmuir 2003, 19, 6778-6787. [CrossRef]

9. Grosch, K.A. The Relation between the Friction and Visco-Elastic Properties of Rubber. Proc. R. Soc. A Math. Phys. Eng. Sci. 1963, 274, 21-39. [CrossRef]

10. Singh, A.K.; Juvekar, V.A. Steady dynamic friction at elastomer-hard solid interface: A model based on population balance of bonds. Soft Matter 2011, 7, 10601. [CrossRef]

11. Schertzer, M.; Iglesias, P. Meta-Analysis Comparing Wettability Parameters and the Effect of Wettability on Friction Coefficient in Lubrication. Lubricants 2018, 6, 70. [CrossRef]

12. Kalin, M.; Polajnar, M. The Effect of Wetting and Surface Energy on the Friction and Slip in Oil-Lubricated Contacts. Tribol. Lett. 2013, 52, 185-194. [CrossRef]

13. Dieterich, J.H. Modeling of rock friction: 1. Experimental results and constitutive equations. J. Geophys. Res. Solid Earth 1979, 84, 2161-2168. [CrossRef]

14. Ruina, A.L. Friction Laws and Instabilities: A Quasistatic Analysis of Some Dry Frictional Behavior; Division of Engineering, Brown University: Providence, RI, USA, 1980. 
15. Putelat, T.; Dawes, J.H.P.; Willis, J.R. On the microphysical foundations of rate-and-state friction. J. Mech. Phys. Solids 2011, 59, 1062-1075. [CrossRef]

16. Briscoe, B. Wear of polymers: An essay on fundamental aspects. Tribol. Int. 1981, 14, 231-243. [CrossRef]

17. Axén, N.; Jacobson, S. A model for the abrasive wear resistance of multiphase materials. Wear 1994, 174, 187-199. [CrossRef]

18. Briscoe, B.J.; Sinha, S.K. Scratch Resistance and Localised Damage Characteristics of Polymer Surfaces-A Review. Materialwiss. Werkstofftech. 2003, 34, 989-1002. [CrossRef]

19. Shipway, P.H.; Ngao, N.K. Microscale abrasive wear of polymeric materials. Wear 2003, 255, 742-750. [CrossRef]

20. Gellman, A.J.; Spencer, N.D. Surface Chemistry in Tribology. In Wear-Materials, Mechanisms and Practice; John Wiley \& Sons Ltd.: London, UK, 2005; pp. 95-122.

21. Persson, B.N.J.; Mugele, F. Squeeze-out and wear: Fundamental principles and applications. J. Phys. Condens. Matter 2004, 16, R295-R355. [CrossRef]

22. Wu-Bavouzet, F.; Clain-Burckbuchler, J.; Buguin, A.; de Gennes, P.G.; Brochard-Wyart, F. Stick-Slip: Wet Versus Dry. J. Adhes. 2007, 83, 761-784. [CrossRef]

23. Bhushan, B. Adhesion and stiction: Mechanisms, measurement techniques, and methods for reduction. J. Vac. Sci. Technol. B 2003, 21, 2262. [CrossRef]

24. Diew, M.; Ernesto, A.; Cayer-Barrioz, J.; Mazuyer, D. Stribeck and Traction Curves Under Moderate Contact Pressure: From Friction to Interfacial Rheology. Tribol. Lett. 2015, 57, 8. [CrossRef]

25. Gebhardt, K.F. Grundlagen der Physikalischen Chemie von Grenzflächen und Methoden zur Bestimmung Grenzflächenenergetischer Probleme; IGB: Paris, France, 1982.

26. Busscher, H.J.; van Pelt, A.W.J.; de Boer, P.; de Jong, H.P.; Arends, J. The effect of surface roughening of polymers on measured contact angles of liquids. Colloids Surfaces 1984, 9, 319-331. [CrossRef]

(C) 2019 by the authors. Licensee MDPI, Basel, Switzerland. This article is an open access article distributed under the terms and conditions of the Creative Commons Attribution (CC BY) license (http:/ / creativecommons.org/licenses/by/4.0/). 